\title{
Toward a New Addressing Scheme for a Service-Centric Internet
}

\author{
A. Martinez, X. Masip-Bruin, W. Ramirez, R. Serral-Gracià, E. Marin-Tordera, M. Yannuzzi \\ Advanced Network Architectures Lab (CRAAX), Technical University of Catalunya (UPC), Spain \\ E-mail: \{annym, xmasip, wramirez, rserral, eva, yannuzzi\}@ac.upc.edu
}

\begin{abstract}
Traditional network architectures based on the Internet Protocol (IP) are now being questioned by the research community, since they are no longer positioned as the most suitable paradigm for supporting the increasing diversity of applications and uses of the Internet. A key issue in this subject is that, although the IP protocol has provided the basis for the rapid evolution of the Internet, its addressing scheme is not prepared to face the challenges posed by many foreseen applications. In light of this, different initiatives worldwide have started specific research programs to address these problems and work toward the "Future Internet". The TARIFA project represents one of these initiatives, and it is positioned as a clean slate alternative aimed at overcoming the critical issues in today's Internet. The novelty in TARIFA resides in the fact that any "commodity" in the network can be composed as a set of atomic services, which can be in turn assembled through a service-centric model for building a promising Internet architecture. In this paper, we focus on the space requirements and set the basis for a new addressing scheme suitable for service-centric network architectures such as the one proposed by TARIFA. The addressing scheme discussed in this paper is general in scope, and could be applied not only to architectures based on the composition of services but also to user and data-centric Internet architectures.
\end{abstract}

Index Terms-Naming and addressing, locators, identifiers, service-centric networks, clean slate, scalability.

\section{INTRODUCTION}

The rapid growth of users and applications that we have witnessed in the last few years is posing complex challenges to the Internet community. To face these issues, multiple research activities were started worldwide, such as GENI [1], 4WARD [2], DONA [3], TRILOGY [4], etc. One such initiative is TARIFA [5], which has developed a clean slate architecture for the Future Internet (FI), where the network paradigm is no longer based on the interconnection of interfaces/machines, but rather on the interconnection of services/resources-an approach that seems to meet much better the requirements of both emerging and future applications. TARIFA takes as a basis a service-centric paradigm, where communications can be composed on the fly and dynamically adapted by assembling reusable components or services.

A major concern, however, in service-centric networks is the addressing scheme used for making possible this shift of paradigm. In the past few years, the design of scalable routing and addressing architectures for the Internet has become the target of numerous research efforts. Concerns related to the scalability of the routing system, and the impending exhaustion of the IPv4 address space have led to important proposals, such as the Locator/IDentifier Separation Protocol (LISP) [6]. The driver for initiatives such as LISP is that current Internet addresses combine two functions: 1) location which represents the point of attachment of a device to the network, i.e., "where" the device is; and 2) identification, indicating "who" the device is. In other words, current IP addresses have dual semantics since locators and identifiers are under the same numeric space. Several papers and reports can be found in the literature claiming that the semantic overloading of IP addresses is putting significant pressure on the capabilities of the routing and the addressing systems [7], since these are starting to hinder the progress of Internet technologies and applications.

In this context, service-centric architectures combined with novel addressing schemes arise as promising alternatives to traditional "OSIfied" IP networks. On this basis, this paper focuses on the space requirements and sets the basis for a new addressing scheme suitable for service-centric network architectures. The addressing scheme discussed in this paper was developed and evaluated as part of the TARIFA project.

The remainder of the paper is organized as follows. Section II overviews the TARIFA concept, while Section III discusses an addressing scheme suitable for service-centric Internet architectures. The addressing scheme is then assessed in Section IV, and finally, Section V concludes the paper.

\section{TARIFA: A SERVICE-CENTRIC ARCHITECTURE FOR THE FUTURE INTERNET}

TARIFA [5] is a research initiative that defines an Internet architecture from scratch by essentially avoiding the traditional hierarchy of network layers. This approach is based on a service-centric paradigm where services become the fundamental functional components. TARIFA enables dynamic composition of services consisting of non-divisible or atomic functions, such that any commodity in the network can be exposed either as an atomic or a composed service. Atomic services refer to well-defined and self-contained functions commonly used in networking protocols (e.g. sequence numbers, flow control, etc.), which are basically used to establish communications for consuming (composed) services. Composed services, on the other hand, are network services or applications with a wider scope than just establishing communications (e.g. a directory service, a printer service, etc), which are assembled as a collection of atomic services.

This newly proposed architecture is context-aware, since communications are built in situ taking into account context variations as well as specific service requirements. More precisely, communications are established by assembling atomic services into workflows, which end up composing a service to obtain the desired functionality and performance constrains. 
In this framework, the network is defined as a set of nodes and services. Nodes are autonomous entities that are capable of providing and consuming services, and thereby provide a natural environment for service composition and execution.

Figure 1 depicts the node architecture proposed in TARIFA. The architectural components are defined so as to fulfill the definition, composition, and provisioning of services under the particularities of the service-centric Internet desired. The proposed architecture has the following features:

- Context-awareness and dynamic adaptability during execution time.

- It is centered on the interconnection of services/resources rather than the interconnection of machines/interfaces.

- Semantic identification and addressing of nodes, resources, and services.

- QoS is integrated into routing and service discovery.

- Users can influence both service choice and routing.

- Security can be natively integrated in the design.

Overall, service-centric approaches are foreseen to provide seamless communications and seem to be better prepared to deal with the expected heterogeneity and dynamics of future networks. However, to make this possible, novel addressing schemes need to be devised. We proceed to describe one possible approach toward this end.

\section{TOWARd A NEW AdDrESSING SCHEME}

In our addressing scheme, we consider locators and identifiers as independent units. The terms "naming space" shall refer to the space of identification of services and resources available in the network. In particular, we use fixed-length bit structures which allow a unique and global identification of services and/or resources. We call these bit structures TIDs, and as we shall show later, they follow a given hierarchy. Our addressing scheme does not entail any kind of restriction

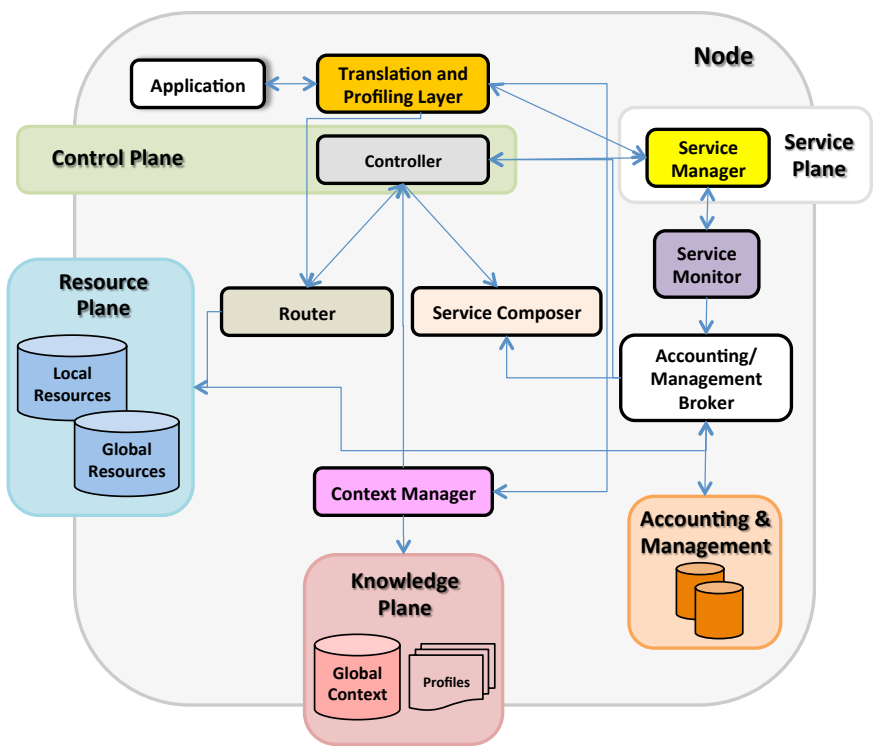

Figure 1. The TARIFA's node architecture (for additional information the reader is referred to TARIFA's Deliverable TWP1.1.D1, "Architecture Definition," February 2011). over "what can be identified"; in other words, both tangible commodities (e.g., a PC, a PDA, etc.) as well as intangible objects (e.g., files, services, applications, etc.) may have their own identifier (TID).

The generic format of a TID is shown in Figure 2. From the figure it is possible to distinguish three fixed size fields, namely:

- Entity: This 64 bit field refers to the root entity instantiating a given service and/or resource. As in current citizen identification systems, where each citizen is identified through a unique number, our approach intends to assign a domain of identification to each citizen, organization, virtual group or enterprise, under which each entity will identify the set or subset of services and resources of its interest.

- Service: This 64 bit field allows to address all available services for a given entity. By this, one entity is capable of addressing a total of $2^{64}$ services, which is actually the square of the addressing space of current Internet.

- Resource: This 32 bit field allows to address all available resources within a given entity or entity/service pair.

A key feature of the proposed identification scheme is its twofold purpose. On one side, it serves to uniquely identify services or resources, and, on the other side, it provides composed identification, meaning that we can identify services related to a specific resource (e.g. a printing service offered by a given printer). It can also be observed that we aim at fixedlength identifiers (160 bits), since in practice it is known to be the best alternative for hardware simplicity and cost. Indeed, carrier-grade network devices are especially designed and tailored to optimize forwarding tasks - for which switching based on fixed length fields is critical. On the other hand, transport networks are increasingly integrating optical solutions, and in this matter, variable length identifiers would imply more electronic processing, which goes against the all optical trend.

Determining the size of the proposed TID is a major challenge, as it must suit the complete space of identifiable objects. Based on the idea of providing entities with an identification block allowing the unique naming of services and resources, we faced the challenge of the number of entities that our space of identifiers shall be able to address. The answer boils down to overestimating the space to be addressed. To this end, assuming that today's worldwide population is around $7 \times 10^{9}$, a total of 33 bits would be required to address the complete human population. According to the United Nations Demographic Forecast [8], the population for 2300 is estimated to be around 8.97 billion, meaning that 34 bits would be enough to globe this figure. Taking in consideration that many other entities (enterprises, organizations, logical groups, and governments) could be provided of a block of identifiers, a gap of 30 extra bits seems to be a suitable number. In this sense, $2^{64}$ entities are addressable on the network. Each entity is able to address $2^{96}$ objects, semantically differentiated in two fields: service (64 bits) and resource (32 bits). The main advantage of such a type of identification is that it allows automatic ID clustering or aggregation.

Note that the proposed solution also gives support to net- 


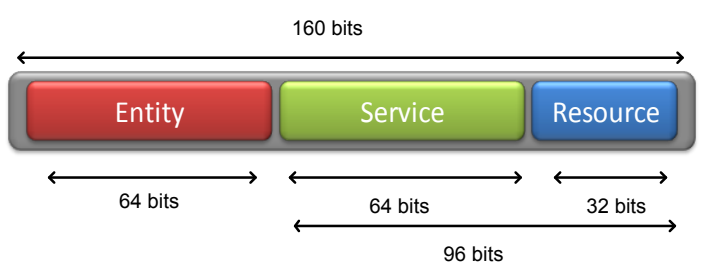

Figure 2. Identifiers: TID format and size for service-centric architectures.

works that lack infrastructure, such as ad hoc or sensor networks. These networks may be internally managed by the manufacturer's identifier, which would not be visible to the global Internet. An aggregation point ruled under our addressing scheme will make the network visible outside its boundaries, and therefore both identification schemes can coexist under the same environment.

Another important aspect of an addressing scheme for service-centric networks is to provide users with the capability of using well-known and/or already defined services, and for this, knowledge on the desired service identifier is required. Since long numeric addresses cannot provide friendly human readable identifiers, service-centric architectures can rely on friendly names enabling users to directly access wellknown services. In TARIFA, these are called TFNIs (TARIFA Friendly Name Identifiers), and they consist of a string based identifier following the same hierarchical structure proposed for the TIDs, that is, a triplet of the form: entity-serviceresource. This means, that services under the same entity will share a common prefix (e.g. companyA.videoServ, companyA.printServ, etc.). To make this possible, the service-centric architecture needs to provide support for solving friendly name identifiers into TIDs, which is typically under the scope of the Domain Name System (DNS).

Due to the separation of the addressing space into two parts, a second group of addresses called TLOCs are considered in our scheme, which compose the location space (see Figure 3). A TLOC is a fixed length bit structure reflecting the location of a resource or service in a numeric format. As it is the case of the TIDs, the proposed TLOCs are globally unique, meaning that they encode addresses over the entire scope of the network. Given the nature of service-centric approaches, locators are not aimed to be only pointers to physical objects, but also to virtual entities. This raises a new challenge related to the mappings for virtual entities (this issue will be discussed further on in Section III-A).

As shown in Figure 3, the proposed TLOCs provide flexibility regarding the location scheme used, allowing the coexistence of different representations, such as flat location schemes, hierarchical schemes, or those based on geolocation, among many others. A fixed number of 8 bits is reserved for the location scheme, while the remaining 88 bits are used for the direct representation of the locators for a given scheme.

In order to ensure that a geolocation scheme under the proposed model can provide the precision and resolution required for a feasible addressing scheme, we have confirmed that an 88 bit locator field can offer very high resolution. Indeed, the total area of the planet's surface is around

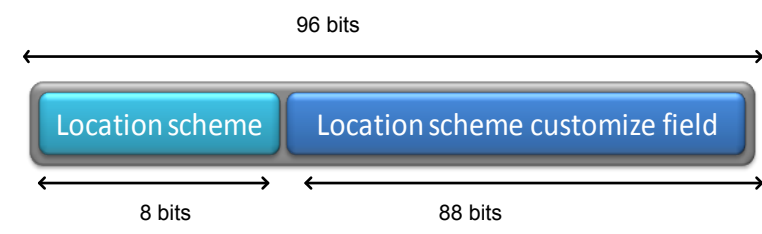

Figure 3. Locators: TLOC format and size for service-centric architectures.

$S_{\text {Earth }}=510.086 \times 10^{9} \mathrm{~m}^{2}$, so for a desired resolution of $1 \mathrm{~mm}^{2}$ (this means being able to locate objects as small as $1 \mathrm{~mm}^{2}$ on the Earth's surface) 69 bits are needed. With 88 bits, this allows resolutions up to the order of $\mathrm{nm}^{2}$, which in the future opens the possibility to locate entities at nanoscales (e.g., in the field of nanonetworking).

\section{A. TID - TLOC mapping.}

With the separation of locators and identifiers in two different spaces, a new concern emerges, which refers to the need of a mechanism able to map identifiers to locators. Many approaches can be found in the recent literature dealing with such a mapping system [9], [10], [11], [12].

In our addressing scheme, the proposed identifier-to-locator mapping is based on DHTs. Similarly to [12], nodes within the mapping system are arranged in a Chord-like ring topology, and are responsible of a group of (key, values) pairs, where keys point to identifiers and values to locators. The advantages of DHT-based implementations for the mapping system are twofold. In the first place, they offer a fully decentralized and easy to maintain system; and second, they show excellent properties in terms of scalability, since, as demonstrated in [11], these schemes experience sublinear growth with the number of addressable nodes $n$. In light of this, a DHT-based mapping system was implemented in the framework of the TARIFA project, in the form of a mapping overlay.

As mentioned above, an important issue related to the mapping of identifiers is how to solve the pointers to virtual objects-note that this functionality does not exist in today's Internet. In TARIFA, we considered two possible approaches:

- By indirect mapping, meaning that, the first retrieved mapping points to the identifier hosting the virtual instance (physical object), followed by a recursive lookup which retrieves the locator for the latter.

- By direct mapping, which means, that the mapping system supports direct relation to locators for virtual object identifiers.

\section{Performance Evaluation}

This section presents an analytical evaluation of the mapping system, with special focus on its scalability. For simplicity, we made the following assumptions. We assume that services are reachable only through one locator (TLOC), and that the mapping entries are uniformly distributed over the mapping system. The analysis aims to examine the amount of entries that can be handled by the mapping system, as a function of the number of mapping nodes and their forwarding and storage capacities. 
As mentioned above, our Chord-like ring is composed of a set of nodes in the form of a mapping overlay, which from now on, we will refer to as Chord Nodes [13]. The number of current entries in the mapping overlay shall be denoted as $T_{T I D S}$, which will be clearly bounded by the total number of possible TIDs in the system, i.e., $1,4615 \times 10^{48}=2^{160}$. This value is obviously reached when the entire space of identifiers has been assigned, and hence all the TIDs must be present in the mapping system.

Under the previous assumptions, any mapping entry consists of a TID-TLOC pair with a total length of 32 bytes (see expression (1)), so the length of an entry in the mapping system, $L_{\text {entry }}$, can be denoted as:

$$
L_{\text {entry }}=T I D_{\text {length }}+\text { TLOC } C_{\text {length }}=256 \text { bits }
$$

Thus, the total number of Chord Nodes needed (denoted as $T_{\text {chord_nodes }}$ ) can be expressed as a function of the total number of identifiers $T_{T I D S}$ currently in the mapping system, and the average storage capacity of the Chord Nodes $\left(S_{\text {chord_nodes }}\right)$ as follows:

$$
T_{\text {chord_nodes }} \geq\left\lceil\frac{32 \text { bytes } T_{T I D S}}{S_{\text {chord_nodes }}}\right\rceil
$$

From this expression, we can notice that there is obviously a trade-off between the total number of nodes conforming the mapping overlay and the storage capabilities of each of these nodes.

Besides the Chord Nodes' storage capacity, the forwarding rate is another important metric for the scalability assessment. Let $R_{\text {chord_nodes }}$ be the number of requests per second that a Chord Node is able to forward. The requests received by the Chord Nodes are essentially update and lookup messages. Update messages aim to introduce new mappings in the system (or update the existing ones, e.g., due to mobility), whilst lookup messages aim at requesting the retrieval of a mapping. In any case, the mappings are always requested for a specific service identifier.

Let $P_{\text {requests }}$ be the percentage of requests per second received on average for each TID. The values used for $P_{\text {requests }}$ are taken from the estimations made in [11] for Internet traffic under a practical environment. Furthermore, in [14] the authors have shown that the average routing path length for a DHT-based Chord Mapping System is:

$$
<\text { Routing Path Length }>=\frac{1}{2} \log \left(T_{\text {chord_nodes }}\right)
$$

Thus, if we assume that the requests are evenly distributed among all Chord Nodes, the lower bound of the forwarding rate of each Chord Node ( $\left.R_{\text {chord_node }}\right)$ can be expressed as a function of the average routing path length and the total number of requests per chord node, that is:

$$
R_{\text {chord_node }}>\frac{1}{2} \log \left(T_{\text {chord_nodes }}\right) \frac{P_{\text {requests }} T_{T I D S}}{T_{\text {chord_nodes }}}
$$

From the above inequality, the maximum number of identifiers that can be used in the mapping system is thus bounded by:

$$
T_{\text {TIDS }}^{\max }=\left\lfloor\frac{2 R_{\text {chord_node }} T_{\text {chord_nodes }}}{\log \left(T_{\text {chord_nodes }}\right) P_{\text {requests }}}\right\rfloor
$$

Expressions (2) and (5) together determine the scaling properties of the mapping system.

\section{A. Evaluation Results}

The results shown in this section are basically an attempt to evaluate our addressing and mapping scheme under currently available technology. It is a fact that networking technologies are pushing toward higher storage capacities and forwarding rates, so in this sense, the addressable space of identifiers will tend to increase as node capabilities evolve. The scalability evaluation is carried out for two different scenarios, which correspond to two typical forwarding rates available with current technology.

For the first scenario, we assume as in [11] that the forwarding rate is $R_{\text {chord_node }}=10^{8}$ packets per second (pps). Figure 4 shows a three-dimensional representation of the size of the identifiers space $\left(T_{T I D S}\right)$, as a function of the percentage of requests in the mapping system and the total number of mapping nodes (Chord Nodes). As shown in Figure 4, the addressable space of identifiers can increase (and so the number of mapping entries in our system) for lower percentages of mapping requests and higher number of Chord Nodes. This is consistent with the fact that the number of identifiers that our system can support is expected to be higher as the incoming requests decrease and the mapping workload can be distributed among more elements in the overlay. From Figure 4 , we also notice that with a structure of mapping elements of barely $3.1 \%$ of all addressable locations, a total of $7.74 \times 10^{35}$ identifiers can be mapped into the system, which is 21 orders of magnitude greater than the base numbers found in [11], and 26 orders of magnitude greater than the addressable space reached with IPv4.

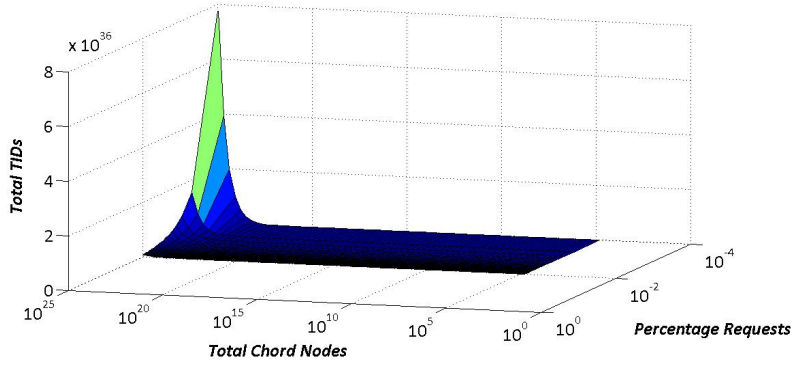

Figure 4. Mapping space as a function of the percentage of requests and the number of Chord Nodes in the system $\left(R_{\text {chord_node }}=10^{8} \mathrm{pps}\right)$. 
For the second scenario of evaluation, we took as a reference the forwarding rate of Juniper's T Series Core Routers [15], being $R_{\text {chord_node }}=30 \times 10^{12} \mathrm{pps}$. As in the previous scenario, Figure 5 depicts the growth of the identifiers space (and thus of the mapping space) as a function of the percentage of mapping requests and total number of Chord Nodes. The idea behind depicting the behavior of the mapping space is to evaluate the tendency upon more challenging capacities.

From the analysis of the results obtained for both scenarios, we conclude that, for a fixed forwarding rate of $30 \times 10^{12} \mathrm{pps}$ and even with less advantageous conditions (e.g., with a high percentage of mapping requests to be resolved and with low number of Chord Nodes), the space of identifiers is $10^{10}$ times the number of persons on Earth. In summary, if the forwarding rate increases to the order of tens of Tera pps, the addressable space can increase by 5 orders of magnitude with respect to the previous scenario.

As it can be observed from inequation (2), the dimension of the mapping space directly affects the storage requirements of the Chord Nodes. Figure 6 depicts the storage requirements per Chord Node for the first scenario $\left(R_{\text {chord_node }}=10^{8} \mathrm{pps}\right)$, as a function of the total number of elements in the mapping system and for different percentages of incoming requests. The figure shows that the storage requirements are around the order of Terabytes, a result that is in line with current available technology, meaning that the chronological evolution of storage technology will positively influence the size of the address space and thus the capacity of the mapping system.

The results obtained from the previous analysis can actually be enhanced by means of two mechanisms: caching and aggregation. Caching would decrease the number of incoming mapping requests into the system, while aggregation would reduce the storage space required for keeping mapping entries within Chord Nodes mapping tables. These mechanisms are out of scope of this work, but will be considered in our future work.

\section{CONCLUSIONS}

In this paper, we have focused on one of the central research challenges of the Future Internet, which is its addressing scheme. We have described a new addressing strategy suitable for service-centric network architectures, which was introduced and assessed in the framework of the TARIFA Project [5]. Our analysis has shown that the proposed scheme can cover the potential requirements of a wide set of applications in the Future Internet, and especially those of service-centric network architectures. Despite these strengths, a number of issues remain unsolved, such the development of a resolution name system, as well as caching and aggregation techniques which can effectively improve the overall performance of the mapping system.

\section{ACKNOWLEDGEMENTS}

The authors would like to acknowledge all colleagues involved in the TARIFA project for their support in the definition of this work. This work was supported in part by the TARIFA project, by the Spanish Ministry of Science and Innovation

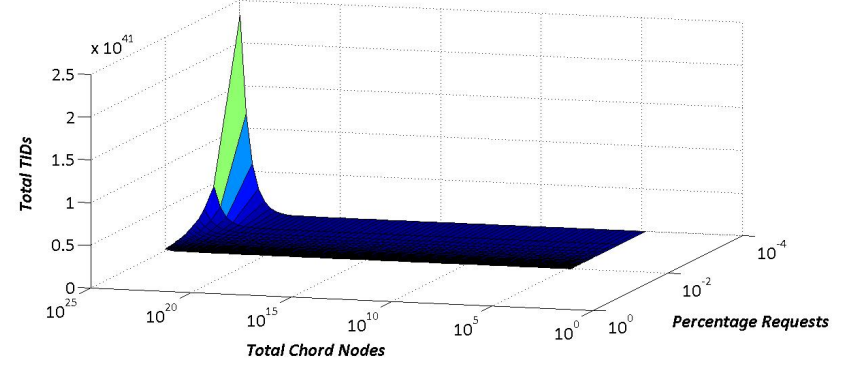

Figure 5. Mapping space as a function of the percentage of requests and the number of Chord Nodes in the system $\left(R_{\text {chord_node }}=30 \times 10^{12} \mathrm{pps}\right)$.

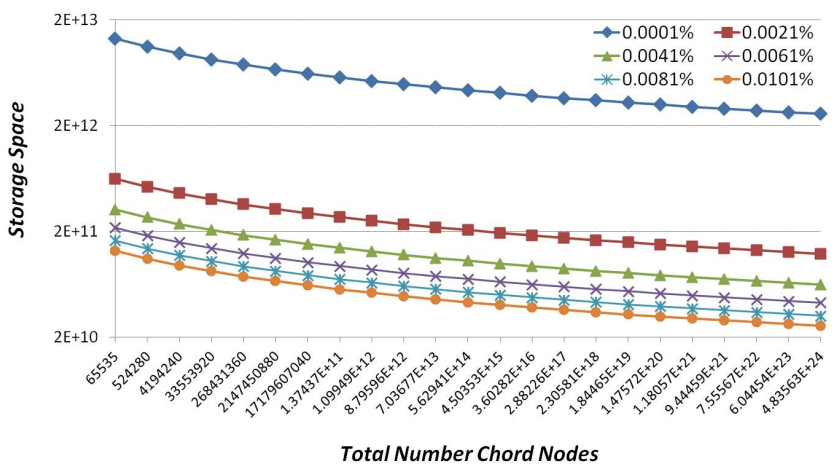

Figure 6. Storage Requirements as a function of the total number of Chord Nodes in the system for different incoming requests (values for the first scenario, i.e., $R_{\text {chord_node }}=10^{8} \mathrm{pps}$ ).

under contract TEC2009-07041, and by the Catalan Research Council (CIRIT) under contract 2009 SGR1508.

\section{REFERENCES}

[1] GENI: http://www.geni.net/.

[2] 4WARD, FP7 EU project: http://www.4ward-project.eu/.

[3] DONA: http://radlab.cs.berkeley.edu/wiki/DONA.

[4] TRILOGY Project: http://trilogy-project.org/.

[5] TARIFA http://www.i2cat.net/en/projecte/tarifa-1.

[6] D. Farinacci et al., "Locator/ID Separation Protocol (LISP)," IETF draft-ietf-lisp-19.txt, January 2012.

[7] D. Meyer et al., "Report from the IAB Workshop on Routing and Addressing," IETF RFC 4984, September 2007.

[8] World Population to 2300, "Official United Nations Estimates and Projections of World, Regional and National Population size, growth and demographic indicators," http://www.un.org/esa/population/publications/longrange2/WorldPop2300final.pdf

[9] V. Fuller et al., "LISP Alternative Topology (LISP+ALT)", IETF draftietf-lisp-alt-10.txt, December 2011.

[10] E. Lear, "NERD: A Not-so-novel EID to RLOC Database", IETF draftlear-lisp-nerd-08.txt, September 2010.

[11] H. Luo, Y. Kin, and H. Zhang, "A DHT-based Identifier-to-locator Mapping Approach for a Scalable Internet," IEEE Transaction on Parallel and Distributed Systems, Vol 20. no 10, October 2009.

[12] K. Lampropoulos and S. Denazis, "Identity Management Directions in Future Internet," IEEE Communications Magazine, Vol 49. no 12, December 2011.

[13] Y. Jimenez and C. Cervello, "TARIFA Deliverable TWP4.2.D1: Design of specific functionalities," April 2011.

[14] G. Cordasco et al., "Degree-Optimal Deterministic Routing for P2P Systems," Proc. of the 10th IEEE Symposium on Computers and Communications (ISCC 2005), Cartagena, Spain, June 2005.

[15] Juniper T-Series Core Router: http://www.juniper.net/us/en/local/pdf/ datasheets/1000051-en.pdf. 